\title{
Drug Therapeutic in the Subacute and Chronic Phase of Chikungunya Virus Infection
}

\author{
Mariana do Socorro Quaresma Silva ${ }^{1}$, Rita Catarina Medeiros Sousa ${ }^{1,2}$, \\ Cezar Augusto Muniz Caldas ${ }^{1}$ \\ ${ }^{1}$ Universidade Federal do Pará, Belém, Pará, Brasil \\ ${ }^{2}$ Universidade Tropical Medicine Nucleus, Belém, Pará, Brasil \\ Email: marianaquaresma@yahoo.com.br,ritaclosset@uol.com.br,cezar_caldas@yahoo.com.br
}

How to cite this paper: Silva, M.S.Q., Sousa, R.C.M. and Caldas, C.A.M. (2021) Drug Therapeutic in the Subacute and Chronic Phase of Chikungunya Virus Infection. Open Journal of Rheumatology and Autoimmune Diseases, 11, 36-47. https://doi.org/10.4236/ojra.2021.112005

Received: February 25, 2021

Accepted: April 19, 2021

Published: April 22, 2021

Copyright $\odot 2021$ by author(s) and Scientific Research Publishing Inc. This work is licensed under the Creative Commons Attribution International License (CC BY 4.0).

http://creativecommons.org/licenses/by/4.0/

\begin{abstract}
Background: Chikungunya fever is an infectious disease that can evolve to a subacute or chronic condition, with changes in the daily activities of patients. Drugs that aim to reduce these symptoms are used, such as corticoids (acute phase) and disease-modifying anti-rheumatic drugs (chronic phase). Objective: To evaluate the clinical response to drug therapy in the subacute and chronic phase of infection by the Chikungunya virus. Methodology: A prospective and a retrospective study with patients with subacute and chronic Chikungunya infection, out at the infection and autoimmunity outpatient clinic at the Nucleus of Tropical Medicine, from January 2016 to December 2019, in the morning of Thursdays. The patient was observed in the Baseline, first and second return, and drugs were introduced according to the stage of the disease with subsequent reassessment. The Visual Analogue Scale (VAS) was applied to all evaluation moments. Results: 101 patients were evaluated, and arthralgia was the predominant symptom in the three evaluated moments. According to the VAS, moderate baseline pain was observed in $58.1 \%$ and $58.6 \%$ of subacute and chronic cases, respectively. On the first return, moderate pain still predominated in $46.2 \%$ in subacute cases and $43 \%$ in chronic cases. In the second visit, all patients were in the chronic phase of the disease, $43.8 \%$ had VAS with no pain. Regarding the number of compromised joints in the Baseline, polyarticular involvement predominated in both subacute (79\%) and chronic $(74.1 \%)$ cases, in the first return, oligoarticular involvement predominated in $53.8 \%$ of subacute cases and $54.7 \%$ in chronic cases and, the second return, $40.6 \%$ of the patients had oligoarticular involvement and $43.8 \%$ had no joint involvement. As for the use of medications in the Baseline, 33.4\% of subacute cases used antiinflammatory drugs, and $40 \%$ of chronic cases used corticosteroids. At the first visit, $25 \%$ of chronic patients were already using combined corticosteroids and methotrexate and $15 \%$ were
\end{abstract}


using only methotrexate. In the second return, $35.1 \%$ used combined methotrexate and corticosteroids, and $64.9 \%$ used only methotrexate. Safety in the use of methotrexate was observed in the context of CHIKV treatment, as the number of adverse reactions was minimal (three patients) and the medication was well tolerated. Conclusion: It was observed that with the adjustment of the medications, there was a reduction in joint impairment, VAS showed mild pain indexes and in some cases with no pain, showing the benefit of using therapy in subacute and chronic cases and improving quality of life of these users.

\section{Keywords}

Chikungunya Fever, Post-Infectious Inflammatory Disease, Alphavirus

\section{Introduction}

Chikungunya fever is a disease caused by the Chikungunya virus, transmitted by the bite of female Aedes aegypti mosquitoes and, less commonly, Aedes albopictus [1]. The Chikungunya virus has two distinct transmission cycles: enzootic and urban [2].

The Chikungunya fever pathophysiology is poorly understood and involves predominantly peripheral mechanisms, however, there is an unrestrained establishment of inflammatory responses, thus characterizing a dysregulation of inflammation in the acute and chronic phase of the disease [3].

After the infected mosquito bite, the incubation period lasts on average 3 to 7 days (range: 1 - 12 days), followed by fever associated with arthralgia/arthritis (87\%), low back pain (67\%), and headache (62\%). Viremia persists up to 8 days after the onset of clinical symptoms and may disappear after acute symptoms, with a resolution of the condition [4].

Some patients may have relapsed rheumatological symptoms (for example, polyarthralgia, polyarthritis, and tenosynovitis) in the months following the acute disease, thus progressing to a subacute phase of the disease that lasts up to three months [5].

Confirmation of recent infection would be given by the following results: 1) Isolation of Chikungunya virus, including identification and confirmation; 2) Detection of Chikungunya virus by real-time Polymerase chain reaction; 3) IgM immunoassay reagent for Chikungunya virus or; 4) Demonstration of seroconversion or a four-fold increase in PRNT, Hemoagglutination or ELISA tests [6].

In the subacute phase of Chikungunya fever, non-hormonal anti-inflammatory drugs and/or adjuvant medications may be used to treat pain (anticonvulsants or antidepressants) in cases refractory to the use of analgesics and opioids. In patients with moderate to severe musculoskeletal pain, or those with contraindications to the use of these medications, the use of prednisone or prednisolone is recommended, at a dose of up to $20 \mathrm{mg}$ per day, the reduction should be made 
slowly and gradually, according to the patient's response [7].

Because of presenting a picture similar to rheumatoid arthritis, disease-modifying antirheumatic drugs are used in the treatment of post-chikungunya arthritis, this phase of the disease being referred to as post-chikungunya chronic inflammatory rheumatism [8].

The treatment approach is based on the fact that the acute phase of chikungunya fever is infectious, but the chronic one is a post-infectious inflammatory disease. Patients with Chikungunya virus infection, who have a musculoskeletal disease and symptoms persisting for more than 3 months should be treated in the same way as patients with rheumatoid arthritis [9]. Because of this context, the following question was established: can the use of methotrexate be used as initial therapy in chronic cases of Chikungunya fever that do not benefit from corticosteroids, or would their adverse events make use difficult?

This study aimed to evaluate the clinical response to drug therapy in the subacute and chronic phase of infection by the Chikungunya virus, by observing the improvement of pain and joint edema by observing the laboratory inflammatory response through the measurement of hemosedimentation speed, after starting drug therapy with the use of corticosteroids or methotrexate in the treatment of chronic arthritis by Chikungunya virus, as well as the observation of safety in the use of methotrexate.

\section{Methods}

A prospective and retrospective, longitudinal, descriptive, and analytical study was carried out. The retrospective study needed to be associated with the prospective study to reach a larger population for the study, at the infection and autoimmunity outpatient clinic at the Nucleus of Tropical Medicine (Pará, Brazil), from January 2016 to December 2019 and the general population (people served with a confirmed serological diagnosis of Chikungunya virus) was equivalent to 150 people when applied exclusion criteria, it resulted in a target population with 101 people. Patients with subacute (one to three months of evolution) and chronic (over three months of evolution) cases, classified in this way according to the time of disease progression, were admitted to the study.

The patients included had more than 30 days of illness, aged over 18 years, of both genders, with laboratory confirmation of Chikungunya virus infection by the ELISA method. Patients excluded or did not fit the inclusion criteria or refusal to participate and did not sign the Free and Informed Consent Form.

The mode of admission of patients to the service was spontaneous demand, referrals from medical professionals who knew the service, referrals from epidemiological surveillance in the face of positive serological results for the assessment and classification of the clinical condition.

Data were collected according to an established protocol or data collection instrument, containing the following variables: sex, age group, ethnicity, etc.

The evaluation of the patients occurred in three moments: Baseline (admission 
to the service), first return (45 days after the baseline), and second return (60 days after the first return).

In each evaluation, a research protocol was applied and through the time of infection and the clinical evolution of the patient so that the therapeutic conduct was instituted and the patient was followed up even after the end of the research phase for those who persisted with symptoms. The Visual Analogue Scale was used in each service, considering the patient's response, where mild pain was given a score of 0 to 2, moderate from 3 to 7 , and severe pain from 8 to 10 , at all times of assessment.

The morning stiffness assessment was evaluated taking into account the patient's report regarding the presence of stiffness or not, that is, stiffness with a duration above 30 minutes was considered present.

Therapeutic management in the subacute phase was based on the guidelines of the Brazilian's Ministry of Health regarding the indication for the use of corticosteroids, however, the time of use was assessed individually according to each person, their evolution, and clinical and pain scale assessment, in this way non-hormonal anti-inflammatory drugs were used in case of mild pain and moderate and severe cases of pain, corticosteroids. The maximum initial dose of corticosteroids was $20 \mathrm{mg}$ per day, being reduced by $5 \mathrm{mg}$ every seven days. In cases of pain resurgence during corticosteroid reduction, a $2.5 \mathrm{mg}$ reduction was performed every 7 days. For patients who had more than 3 months of symptoms (chronic), what is recommended by the Brazilian Society of Rheumatology followed, use of methotrexate started at the minimum initial dose of $10 \mathrm{mg}$ per week, with reevaluation of treatment being done every four weeks, with the gradual increase in a dose up to a maximum of $25 \mathrm{mg}$ per week in case of partial response [4].

Thus, methotrexate was used in an initial dose of $10 \mathrm{mg}$, with a progressive increase to $25 \mathrm{mg}$ in case of no clinical improvement, initially associated with corticosteroids, at the same dosage as the subacute phase, but with an early withdrawal proposal, because despite the recommendation of the use of chloroquine by the Brazilian' Ministry of Health, several studies point out, its little effectiveness, for this reason, we will not use it in this study, as treatment of the first choice.

Follow-up was carried out with two returns, the first return after 45 of the initial procedure, and the second return 60 days after the first return. However, some patients, even after the end of the evaluation, continued to follow up because the symptoms persist.

The collected information was stored in a database prepared in a Microsoft

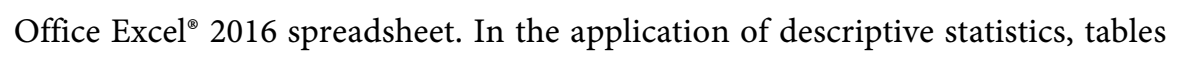
and graphs were constructed to present the results and the position measures were calculated as an arithmetic mean and standard deviation. Analytical statistics were used to evaluate the results of the sample's categorical variables using the Chi-Square Adherence Test for univariate tables, and Partition for variables with multiple categories. Descriptive statistics were performed using the BioEs- 
tat $^{\oplus} 5.4$ software. For decision-making, the significance level $\alpha=0.05$ or $5 \%$ was adopted, signaling the significant values with an asterisk $\left(^{*}\right)$.

\section{Results}

This study included the evaluation of 101 patients, where it was found that $29.7 \%(n=30)$ were in the age group of 50 to 59 years, $89.1 \%(n=90)$ were women, the color of brown skin predominated in 75.2\% $(n=76)$ (Table 1).

Arthralgia was the most observed symptom in all assessments, both in patients with the subacute form and in patients with the chronic form, followed by arthritis, lower limb edema, alopecia, and tendonitis with a gradual reduction of all symptoms in subsequent assessments (Table 2 ).

Table 1. General characteristics of patients with subacute or chronic infection by the Chikungunya virus.

\begin{tabular}{cccc}
\hline Patient data & $\mathrm{n}=101$ & $\%$ & p-value \\
\hline Age group (in years) & 17 & 16.8 & $\mathbf{0 . 0 0 3 6}^{*}$ \\
$<40$ & 26 & 25.7 & \\
40 to 49 & 30 & 29.7 & \\
50 to 59 & 21 & 20.8 & \\
60 to 69 & 7 & 6.9 & \\
$>$ or equal to 70 & $20 / 52.0 \pm 12.5 / 90$ & $<0.0001^{*}$ \\
\hline Minimum/Mean \pm Standard deviation/Maximun age & & & \\
Gender & 90 & 89.1 & \\
Female & 11 & 10.9 & \\
Male & & & $<0.0001^{*}$ \\
Ethnicity & 76 & 75.2 & \\
Brown & 25 & 24.8 & \\
White & & & \\
\hline
\end{tabular}

*Chi-square adherence test. Source: Research protocol.

Table 2. Signs and symptoms in subacute or chronic infection by the Chikungunya virus.

\begin{tabular}{|c|c|c|c|c|c|c|c|}
\hline \multirow[t]{2}{*}{ Signs and symptoms } & \multicolumn{2}{|c|}{$\begin{array}{l}\text { Baseline } \\
(n=101)\end{array}$} & \multicolumn{2}{|c|}{$\begin{array}{c}1^{\circ} \text { return } \\
(n=99)\end{array}$} & \multicolumn{2}{|c|}{$\begin{array}{c}2^{\circ} \text { return } \\
(n=64)\end{array}$} & \multirow{2}{*}{$\mathrm{p}$-value } \\
\hline & $\mathrm{n}$ & $\%$ & $\mathrm{n}$ & $\%$ & $\mathrm{n}$ & $\%$ & \\
\hline Arhralgia & 99 & 98.0 & 83 & 83.8 & 36 & 56.3 & $0.0037^{\star}$ \\
\hline Arthritis & 87 & 86.1 & 43 & 43.4 & 12 & 18.8 & \\
\hline Lower limb edema & 50 & 49.5 & 27 & 27.3 & 5 & 7.8 & \\
\hline Alopecia & 6 & 5.9 & 2 & 2.0 & - & - & \\
\hline Tendonitis & 2 & 2.0 & - & - & - & - & \\
\hline Others & - & - & 5 & 5.1 & 2 & 3.1 & \\
\hline
\end{tabular}

${ }^{\star}$ Chi-square partition test. Source: Research protocol. 
According to the Visual Analogue Scale, moderate pain was observed at baseline in $58.1 \%(n=25)$ of subacute cases and $58.6 \%(n=34)$ of chronic cases. When assessed at the first visit, moderate pain still predominated in $46.2 \%(\mathrm{n}=$ 6) of subacute cases and $43 \%(n=37)$ of chronic cases, in the evaluation during the second visit, where all patients were chronic 43, 8\% $(\mathrm{n}=28)$. Had Visual Analogue Scale with no pain (Table 3).

Joint impairment, determined by counting compromised joints, showed in patients admitted to the service (baseline), whether in the subacute or chronic phase, a polyarticular impairment $79.0 \%(\mathrm{n}=34)$ and $74.1 \%(\mathrm{n}=43)$, respectively. Oligoarticular involvement was predominant in the first return in $53.8 \%$ $(n=7)$ of subacute cases and in $54.7 \%(n=47)$ of chronic cases. In the second assessment, where the group was composed only of patients in the chronic phase, we observed a percentage of patients without arthralgia (43.8\%) (Table 4).

The presence of morning stiffness at baseline was observed in $30.2 \%(n=13)$ of subacute patients and $37.9 \%(n=22)$ of chronic cases. In the first, morning stiffness was not reported by patients in the subacute phase, with this symptom being observed in chronic patients in the first evaluation in $17.4 \%(\mathrm{n}=15)$ and the second evaluation in 9.4\% $(\mathrm{n}=6)$, as shown in Table 5 .

Table 3. Visual analog pain scale in subacute infection or chronic by the Chikungunya virus.

\begin{tabular}{|c|c|c|c|c|c|c|c|c|c|c|}
\hline \multirow{3}{*}{ Pain scale } & \multicolumn{4}{|c|}{ Baseline $(n=101)$} & \multicolumn{4}{|c|}{$1^{\circ}$ return $(\mathrm{n}=99)$} & \multirow{2}{*}{\multicolumn{2}{|c|}{$\begin{array}{c}2^{\circ} \text { return }(\mathrm{n}=64) \\
\begin{array}{c}\text { Chronic } \\
(\mathrm{n}=64)\end{array}\end{array}$}} \\
\hline & \multicolumn{2}{|c|}{$\begin{array}{l}\text { Subacute } \\
(n=9)\end{array}$} & \multicolumn{2}{|c|}{$\begin{array}{l}\text { Chronic } \\
(\mathrm{n}=58)\end{array}$} & \multicolumn{2}{|c|}{$\begin{array}{l}\text { Subacute } \\
(\mathrm{n}=13)\end{array}$} & \multicolumn{2}{|c|}{$\begin{array}{l}\text { Chronic } \\
(n=86)\end{array}$} & & \\
\hline & $\mathrm{n}$ & $\%$ & $\mathrm{n}$ & $\%$ & $\mathbf{n}$ & $\%$ & $\mathbf{n}$ & $\%$ & $\mathbf{n}$ & $\%$ \\
\hline Absent & - & - & - & - & 1 & 7.7 & 13 & 15.1 & 28 & 43.8 \\
\hline Mild & 1 & 2.3 & 5 & 8.6 & 4 & 30.8 & 25 & 29.1 & 18 & 28.1 \\
\hline Moderate & 25 & 58.1 & 34 & 58.6 & 6 & 46.2 & 37 & 43.0 & 14 & 21.9 \\
\hline Severe & 17 & 39.5 & 19 & 32.8 & 2 & 15.4 & 10 & 11.6 & 4 & 6.3 \\
\hline
\end{tabular}

Source: Research protocol.

Table 4. Joint impairment in patients with subacute or chronic infection by the Chikungunya virus.

\begin{tabular}{|c|c|c|c|c|c|c|c|c|c|c|}
\hline \multirow{3}{*}{ Joint } & \multicolumn{4}{|c|}{ Baseline $(n=101)$} & \multicolumn{4}{|c|}{$1^{\circ}$ return $(n=99)$} & \multirow{2}{*}{\multicolumn{2}{|c|}{$\begin{array}{c}2^{\circ} \text { return }(n=64) \\
\begin{array}{c}\text { Chronic } \\
(n=64)\end{array}\end{array}$}} \\
\hline & \multicolumn{2}{|c|}{$\begin{array}{l}\text { Subacute } \\
(n=43)\end{array}$} & \multicolumn{2}{|c|}{$\begin{array}{l}\text { Chronic } \\
(n=58)\end{array}$} & \multicolumn{2}{|c|}{$\begin{array}{l}\text { Subacute } \\
(n=13)\end{array}$} & \multicolumn{2}{|c|}{$\begin{array}{l}\text { Chronic } \\
(n=86)\end{array}$} & & \\
\hline & $\mathrm{n}$ & $\%$ & $\mathbf{n}$ & $\%$ & $\mathbf{n}$ & $\%$ & $\mathbf{n}$ & $\%$ & $\mathrm{n}$ & $\%$ \\
\hline Without arthralgia & - & - & - & - & 1 & 7.7 & 13 & 15.1 & 28 & 43.8 \\
\hline Monoarticular & 2 & 4.7 & - & - & - & - & - & - & - & - \\
\hline Oligoarticular & 7 & 16.3 & 15 & 25.9 & 7 & 53.8 & 47 & 54.7 & 26 & 40.6 \\
\hline Polyarticular & 34 & 79.0 & 43 & 74.1 & 5 & 38.5 & 26 & 30.2 & 10 & 15.6 \\
\hline
\end{tabular}

Source: Research protocol. 
Table 5. Morning stiffness in patients with subacute or chronic infection by the Chikungunya virus.

\begin{tabular}{ccccccccccc}
\hline & \multicolumn{3}{c}{ Baseline $(\mathrm{n}=101)$} & \multicolumn{2}{c}{$1^{\bullet}$ return $(\mathrm{n}=99)$} & $2^{\circ}$ return $(\mathrm{n}=64)$ \\
\cline { 2 - 10 } Morning stiffness & $\begin{array}{c}\text { Subacute } \\
(\mathrm{n}=43)\end{array}$ & $\begin{array}{c}\text { Chronic } \\
(\mathrm{n}=58)\end{array}$ & $\begin{array}{c}\text { Subacute } \\
(\mathrm{n}=13)\end{array}$ & $\begin{array}{c}\text { Chronic } \\
(\mathrm{n}=86)\end{array}$ & $\begin{array}{c}\text { Chronic } \\
(\mathrm{n}=64)\end{array}$ \\
\cline { 2 - 11 } & $\mathrm{n}$ & $\%$ & $\mathrm{n}$ & $\%$ & $\mathrm{n}$ & $\%$ & $\mathrm{n}$ & $\%$ & $\mathrm{n}$ & $\%$ \\
\hline Presence & 13 & 30.2 & 22 & 37.9 & - & - & 15 & 17.4 & 6 & 9.4 \\
Absent & 30 & 79.0 & 36 & 62.1 & 13 & 100.0 & 71 & 82.6 & 58 & 90.6 \\
\hline
\end{tabular}

Source: Research protocol.

The non-specific laboratory evaluation performed did not show any statistically significant result, even in the tests of inflammatory function, however, we emphasize that some respondents did not return with the laboratory results, being a limiting factor in this analysis.

Before undergoing care, it was observed that patients used pain control medications. At baseline, 37.1\% $(\mathrm{n}=13)$ of patients in the subacute phase used analgesics and $40 \%(n=18)$ of chronic patients used corticosteroids. In the first return visit, $75 \%(\mathrm{n}=9)$ of subacute patients and $58.6 \%(\mathrm{n}=41)$ of chronic cases used corticosteroids. In the second return, $61.5 \%(n=24)$ of the patients used methotrexate (Table 6). The methotrexate dosage used, with good clinical response, was $10 \mathrm{mg}$ per week for most patients at all times evaluated. At each evaluation (first return and second return), some patients returned without using the medication, either due to treatment abandonment or due to the end of the prescribed medication cycle.

The time of using methotrexate varied from one to twelve months, where 50\% of patients used it for six months. Tolerability to use was reported by the vast majority, with four (04) people discontinuing the drug on the first return, due to adverse gastric effect, nausea, and vomiting (2), hives (1), worsening arthralgia (1). The use of the drug ensured safety and few adverse events, only nausea was reported, but such events were only in the initial doses. Patients who used it for less than 6 months, except for those who had drug intolerance, were due to clinical improvement. By the end of the study, $36.6 \%$ of patients were discharged from the clinic with remission of symptoms.

\section{Discussion}

Chikungunya infection is an endemic disease in the northern region of Brazil and constitutes an important public health problem, because of the evolution to the subacute and chronic forms, with functional limitations, and difficulty in carrying out daily activities. When analyzing the signs and symptoms, there was a predominance of arthralgia in the three moments analyzed, followed by arthritis, lower limb edema, and alopecia, with gradual reduction of all symptoms in subsequent evaluations.

It is important to note that there was a reduction in the population evaluated in the first and second visits due to the improvement of the clinical condition 
Table 6. Medication use of patients with subacute or chronic infection with the Chikungunya virus.

\begin{tabular}{|c|c|c|c|c|c|c|c|c|c|c|c|}
\hline \multirow{3}{*}{ Medication } & \multicolumn{4}{|c|}{$\begin{array}{l}\text { Baseline } \\
(n=101)\end{array}$} & \multicolumn{4}{|c|}{$\begin{array}{c}1^{\circ} \text { return } \\
(n=99)\end{array}$} & \multirow{2}{*}{\multicolumn{2}{|c|}{$\begin{array}{l}2^{\circ} \text { return } \\
(\mathrm{n}=64)\end{array}$}} & \multirow{3}{*}{ p-value } \\
\hline & \multicolumn{2}{|c|}{$\begin{array}{c}\text { Subacute } \\
(n=43)\end{array}$} & \multicolumn{2}{|c|}{$\begin{array}{l}\text { Chronic } \\
(n=58)\end{array}$} & \multicolumn{2}{|c|}{$\begin{array}{l}\text { Subacute } \\
(n=13)\end{array}$} & \multicolumn{2}{|c|}{$\begin{array}{l}\text { Chronic } \\
(\mathrm{n}=86)\end{array}$} & & & \\
\hline & $\mathbf{n}$ & $\%$ & $\mathbf{n}$ & $\%$ & $\mathbf{n}$ & $\%$ & $\mathrm{n}$ & $\%$ & $\mathbf{n}$ & $\%$ & \\
\hline \multicolumn{12}{|l|}{ Use } \\
\hline Yes & 35 & 81.4 & 45 & 77.6 & 12 & 92.3 & 70 & 81.4 & 39 & 60.9 & \\
\hline No & 8 & 18.6 & 13 & 22.4 & 1 & 7.7 & 16 & 18.6 & 25 & 39.1 & \\
\hline Type & \multicolumn{2}{|c|}{$\mathrm{n}=35$} & \multicolumn{2}{|c|}{$\mathrm{n}=45$} & \multicolumn{2}{|c|}{$\mathrm{n}=12$} & \multicolumn{2}{|c|}{$\mathrm{n}=70$} & \multicolumn{2}{|c|}{$\mathrm{n}=39$} & 0.0203 \\
\hline Analgesic & 13 & 37.1 & 19 & 42.2 & - & - & - & - & - & - & \\
\hline Analgesic + NSAID & 3 & 8.6 & 5 & 11.1 & - & - & - & - & - & - & \\
\hline NSAID & 10 & 28.6 & 3 & 6.7 & 1 & 8.3 & 3 & 4.3 & - & - & \\
\hline Corticoid & 7 & 20.0 & 18 & 40.0 & 9 & 75.0 & 41 & 58.6 & 2 & 5.1 & \\
\hline Corticoid + NSAID & 2 & $5.7 \%$ & 1 & 2.2 & - & - & - & - & - & - & \\
\hline Corticoid + methotrexate & - & - & - & - & 1 & 8.3 & 18 & 25.7 & 13 & 33.4 & \\
\hline Methotrexate & - & - & 1 & 2.2 & 1 & 8.3 & 11 & 15.7 & 24 & 61.5 & \\
\hline
\end{tabular}

${ }^{*}$ Chi-square partition test. NSAID: Non-hormonal anti-inflammatory drugs. Source: Research protocol.

and absenteeism in the visits, however, some patients were rescued through telephone contact and a new evaluation schedule.

During a follow-up of patients with acute Chikungunya fever, some progressed to a subacute and chronic phase and presented a predominant clinical picture of arthralgia and arthritis in both phases, but there was a decrease in pain with the overtime [10], in agreement with this study. Arthralgia that presents in the subacute and chronic phases has a linear downward tendency in the level of pain [11], a fact observed in this study, where the majority had a gradual reduction in pain at each assessment, with evidence through the Visual Analog Pain Scale.

Chronic symptoms are known to decrease over time after initial infection, from $88 \%$ to $100 \%$ during the first six weeks and less than $50 \%$ after three to five years, with variable results depending on the study. The time to complete recovery is still uncertain and some infected individuals remain symptomatic six to eight years after the initial infection [12].

In baseline, all patients had some degree of joint impairment, predominantly polyarticular in subacute and chronic cases, with a reduction in the number of compromised (oligoarticular) joints in the first return, both in patients with subacute symptoms, as in chronic cases, on the second return, when all subjects were in the chronic phase, most patients were asymptomatic, that is, the absence of arthralgias. Usually, the involvement is polyarticular and symmetrical, but it can be asymmetrical and monoarticular [13]. 
In arthritis associated with Chikungunya fever, the levels of rheumatoid factor and anti-cyclic citrullinated peptide antibody are not high, however, it is essential to emphasize that studies show that one-third of patients with Chikungunya virus meets the criteria of the American College of Rheumatology for the diagnosis of rheumatoid arthritis, for example, this is the importance of carrying out such tests [14], as the outpatient clinic in this study does not have a specialized laboratory to perform tests such as rheumatoid factor, anti-cyclic citrullinated peptide, in this research, only $2 \%$ of patients were able to perform these tests, with results within the standard normality.

Subacute cases after the initial assessment and adjustment of therapy benefited from the use of corticosteroids at a maximum dose of $20 \mathrm{mg}$ per day, from a percentage of corticosteroid use of $20 \%$ found in the baseline to $75 \%$ of patients in the first assessment.

The Brazilian Ministry of Health's recommendations were not used because, in addition to the maximum dose being very high $(40 \mathrm{mg})$, the study carried out by the Brazilian Society of Rheumatology did not show benefits in doses above $20 \mathrm{mg}$. The Ministry of Health places a maximum treatment time of 21 days with a gradual dose reduction, disregarding the reduction of symptoms, clinical improvement, and options in case of a therapeutic failure within the specified time [4].

The ideal time of corticosteroid use is also related to dependence or resistance to corticosteroids beyond the third month after the onset of the disease (chronic phase) and is highly suggestive of residual chronic inflammatory rheumatism. When it occurs, the therapeutic handling starts with the use of disease-modifying antirheumatic drugs, such as methotrexate (applied in the treatment of classic rheumatoid arthritis), to control the inflammatory process, prevent bone erosions, and avoid side effects of prolonged corticosteroid therapy [15].

International guidelines recommend methotrexate as a first-line treatment in the treatment of patients with classic rheumatoid arthritis and psoriatic polyarthritis, so as there is no definition of the effectiveness of different disease-modifying antirheumatic drugs, the use of methotrexate is based on the fact that the pathophysiology is similar to rheumatoid arthritis [16].

Despite the lack of studies comparing the effectiveness of methotrexate and hydroxychloroquine in chikungunya fever, in this study methotrexate was used in the treatment of the chronic phase, as the first choice, due to its known anti-inflammatory effects in the control of arthritis.

Methotrexate was introduced to a patient in the chronic phase of the disease. Thus, the recommended initial dose of $10 \mathrm{mg}$ per week was initiated and in cases of refractory response in the subsequent evaluation, it could be increased to 15 $\mathrm{mg}$ per week, up to the maximum dose of $25 \mathrm{mg}$ per week. In the first return, most patients benefited from the minimum dosage (10 mg per week) of methotrexate, in the second return the response to use was reevaluated and $54.2 \%$ of the patients persist using the minimum dose and $41.7 \%$ needed higher doses (15 mg per week) to have a satisfactory response and a small percentage benefited 
from higher doses (20 mg per week) of methotrexate. The use of methotrexate, at an average dose of $15 \mathrm{mg}$ per week, has a benefit in inflammatory rheumatic polyarthritis developed after Chikungunya fever [13]. In this study, the evidenced dose of benefit was between 10 and $15 \mathrm{mg}$ per week

The use of methotrexate associated with corticosteroids was evidenced in this study, observing that in the first return the minimum dose of corticoid used was $10 \mathrm{mg}$ and in the second return the minimum dose used was $5 \mathrm{mg}$, however despite this association, the objective was to reduce the use of corticosteroids to the lowest dose necessary for pain relief, thus, in the second return, a large number of patients using only methotrexate were observed, but some patients whom they persisted with an active clinical picture, benefited from the use of small doses (between 5 to $7.5 \mathrm{mg}$ per day) of corticosteroids with gradual reduction of dosage.

In this study, the time of using methotrexate varied from one to twelve months, where $50 \%$ of the patients used it for six months, with good tolerance to use, but there are few published data to define the time of using methotrexate in post-chikungunya chronic inflammatory rheumatism, the parameter for discontinuing or withdrawing treatment is remission of the condition and laboratory improvement of inflammatory markers [16].

\section{Conclusions}

This study concluded that within the participants the predominant symptom was arthralgia, followed by arthritis. It was also observed that throughout the follow-up period, signs and symptoms (mainly arthralgia and arthritis) tend to regress, not necessarily with a parallel reduction in the values of laboratory tests, and may reflect the natural history of the disease, however, this symptom may be better tolerated with the use of medications.

The evaluation through the hemosedimentation speed did not show a significant result, highlighting the great difficulty of carrying out laboratory tests by the respondents.

The most used drugs were analgesics (in the baseline), corticosteroids (in the first return), and methotrexate (in the second return), compatible with the recommended sequence in the literature for the treatment of Chikungunya fever in its different phases.

Safety in the use of methotrexate was observed in the context of Chikungunya fever treatment, as the number of adverse reactions was minimal (four patients) and the medication was well tolerated.

There was a clinical improvement with a reduction in the number of compromised joints, a reduction in the level of pain, assessed by Visual Analog Pain Scale, after the institution of treatment based on the evolutionary phase of the disease, observing a gradual improvement with each assessment. Dosing between 10 and $15 \mathrm{mg}$ per week was sufficient to control the clinical picture. The time of use varied from one to 12 months, with an average of 6 months of use. 
Thus, in the baseline, first and second return, we observed that with the adjustment of the medications, the number of painful or swollen joints decreases, the Visual Analog Pain Scale reduces to mild pain rates and in some cases with no pain, showing the benefit of using the therapy in subacute and chronic cases and improving the quality of life of these users.

It is hoped, therefore, that the present study can contribute as a source for further studies on the therapy used for Chikungunya infection, aiming at improving the clinic and the quality of life of these patients.

\section{Conflicts of Interest}

The authors declare no conflicts of interest regarding the publication of this paper.

\section{References}

[1] Brasil. Ministério da Saúde (2018) Boletim Epidemiológico, n. 34: Monitoramento dos casos de dengue, febre chikungunya e doença aguda pelo vírus Zika até a semana epidemiológica 30 de 2018.

https://antigo.saude.gov.br/images/pdf/2018/agosto/21/Publicacao-BE-2018-SE-30. pdf

[2] Caglioti, C., et al. (2013) Chikungunya Virus Infection: An Overview. New Microbiologica, 36, 211-227.

[3] Ng, L.F.P. (2017) Immunopathology of Chikungunya Virus Infection: Lessons Learned from Patients and Animal Models. Annual Review of Virology, 4, 413-427. https://doi.org/10.1146/annurev-virology-101416-041808

[4] Marques, C.D.L., et al. (2017) Recomendações da Sociedade Brasileira de Reumatologia para diagnóstico e tratamento da febre chikungunya Parte 2. The Revista Brasileira de Reumatologia, 57, 43851.

[5] Ferreira, C.A., et al. (2019) Beyond Members of the Flaviviridae Family, Sofosbuvir Also Inhibits Chikungunya Virus Replication. Antimicrobial Agents and Chemotherapy, 63, e01389-18. https://doi.org/10.1128/AAC.01389-18

[6] Javelle, E., et al. (2015) Specific Management Fpost-Chikungunya Rheumatic Disorders: A Retrospective Study of 159 Cases in Reunion Island from 2006-2012. PLOS Neglected Tropical Diseases, 9, e0003603. https://doi.org/10.1371/journal.pntd.0003603

[7] Marimoutou, C., et al. (2015) Chikungunya Infection: Self-Reported Rheumatic Morbidity and Impaired Quality of Life Persist 6 Years Later. Clinical Microbiology and Infection, 21, 688-693. https://doi.org/10.1016/j.cmi.2015.02.024

[8] Goupil, B.A. and Mores, C.N. (2016) A Review of Chikungunya Virus-Induced Arthralgia: Clinical Manifestations, Therapeutics, and Pathogenesis. The Open Rheumatology Journal, 10, 129-140. https://doi.org/10.2174/1874312901610010129

[9] Perret, C., et al. (2018) Chikungunya, enfermedad emergente en América Latina. Descripción de los primeros casos en Chile. Revista Chilena de Infectologia, 35, 413-419. https://doi.org/10.4067/s0716-10182018000400413

[10] Sánchez, J.S., Cañón, A.M. and Lombo, J.C. (2019) Subacute and Chronic Symptoms of Chikungunya Fever in a Group of Adults in Colombia. Síntomas subagudos y crónicos de la fiebre de chikungunya en un grupo de personas adultas en Ibagué, Colombia. Biomedica, 39, 587-594. 
https://doi.org/10.7705/biomedica.4350

[11] Castro, A.P.C.R., Lima, R.A. and Nascimento, J.S. (2016) Chikungunya: A visão do clínico de dor. Revista Dor, 17, 299-302.

https://doi.org/10.5935/1806-0013.20160093

[12] Brasil. Ministério da Saúde (2017) Boletim Epidemiológico, v. 48, n. 38-Monitoramento dos casos de dengue, febre de chikungunya e febre pelo vírus Zika até a Semana Epidemiológica 50, 2017.

https://portalarquivos2.saude.gov.br/images/pdf/2018/janeiro/10/2017-046-Publicac ao.pdf

[13] Manimunda, S.P., et al. (2010) Clinical Progression of Chikungunya Fever during Acute and Chronic Arthritic Stages and the Changes in Joint Morphology as Revealed by Imaging. Transactions of the Royal Society of Tropical Medicine and Hygiene, 104, 392-399. https://doi.org/10.1016/j.trstmh.2010.01.011

[14] Foissac, M., et al. (2015) Post-Chikungunya Rheumatoid Arthritis, Saint Martin. Emerging Infectious Diseases, 21, 530-532. https://doi.org/10.3201/eid2103.141397

[15] Chaaithanya, I.K., et al. (2014) Chronic Inflammatory Arthritis with Persisting Bony Erosions in Patients Following Chikungunya Infection. Indian Journal of Medical Research, 140, 142-145.

[16] Cunha, R.V. and Trinta, K.S. (2017) Chikungunya Virus: Clinical Aspects and Treatment-A Review. Memórias do Instituto Oswaldo Cruz, 112, 523-531. https://doi.org/10.1590/0074-02760170044 